\title{
Interventional cardiology in Poland in 2020 - impact of the COVID-19 pandemic. Annual summary report of the Association of Cardiovascular Interventions of the Polish Cardiac Society and Jagiellonian University Medical College*
}

\author{
Zbigniew Siudak ${ }^{1}$, Dariusz Dudek ${ }^{2}$, Marek Grygier ${ }^{3}$, Aleksander Araszkiewicz ${ }^{3}$, Maciej Dąbrowski ${ }^{4}$, \\ Jacek Kusa ${ }^{5}$, Michat Hawranek ${ }^{6}$, Zenon Huczek ${ }^{7}$, Paweł Kralisz ${ }^{8}$, Tomasz Roleder ${ }^{9}$, Wojciech Wojakowski ${ }^{10}$, \\ Radosław Parma ${ }^{10}$, Krzysztof P. Malinowski' ${ }^{2}$ Stanisław Bartuś ${ }^{2}$ \\ ${ }^{1}$ Collegium Medicum, Jan Kochanowski University, Kielce, Poland \\ ${ }^{2}$ Institute of Cardiology, Jagiellonian University Medical College, Krakow, Poland \\ ${ }^{3} 1^{\text {st }}$ Department of Cardiology, Poznan University of Medical Sciences, Poznan, Poland \\ ${ }^{4}$ Department of Interventional Cardiology and Angiology, Institute of Cardiology, Warsaw, Poland \\ ${ }^{5}$ Pediatric Cardiology Department, Regional Specialist Hospital - Research and Development Centre, Wroclaw, Poland \\ ${ }^{6} 3^{\text {rd }}$ Department of Cardiology, Faculty of Medical Sciences in Zabrze, Medical University of Silesia in Katowice, Poland \\ ${ }^{7} 1^{\text {st }}$ Department of Cardiology, Medical University of Warsaw, Warsaw, Poland \\ ${ }^{8}$ Department of Invasive Cardiology, Medical University of Bialystok, Bialystok, Poland \\ ${ }^{9}$ Regional Specialist Hospital, Research and Development Center, Wroclaw, Poland \\ ${ }^{10}$ Department of Cardiology and Structural Heart Diseases, $3^{\text {rd }}$ Division of Cardiology, Medical University of Silesia, Katowice, Poland \\ *This paper presents the analysis of individual procedural data from 154 interventional cardiology centers in Poland that reported their procedures to the \\ ORPKI database in 2020. With regard to NFZ data, we estimate that as many as $90 \%$ of all interventional cardiology procedures in Poland are reported to \\ ORPKI by these 154 of 163 existing centers.
}

As we have previously reported, the ORPKI electronic data capture is monitored continuously and modified according to the needs of the rapidly changing everyday practice of interventional cardiology not only in Poland but also worldwide as required by ESC guidelines [1, 2]. The database is endorsed by the Association of Cardiovascular Interventions of the Polish Cardiac Society (AISN PTK) and is daily operated by the Jagiellonian University Medical College, with currently 154 interventional cardiology centers in Poland reporting. ORPKI database analysis of various trends and patterns is published each year [3-5]. On $31^{\text {st }}$ of December 2020 there were $575 \mathrm{PCl}$ operators certified by the AISN PTK in Poland.

According to the current analysis of the ORPKI database, in comparison to 2019 , there was a substantial $25 \%$ decrease in the total number of reported coronary angiographies (CAG) [1]. There were 130662 CAG, which corresponds to 3394 per 1 million inhabitants per year in 2020 (Figure 1). The distribution of primary diagnosis as the indication for CAG was: 13\% ST-elevation myocardial infarction (STEMI), 16\% non-ST-elevation myocardial infarction (NSTEMI), $27 \%$ unstable angina (UA), and $45 \%$ stable angina (a 5\% increase in acute coronary syndromes (ACS) preponderance that year). The radial approach was utilized in $89 \%$ of all CAG cases, which is a $1 \%$ rise in comparison to 2019 (in STEMI patients, 85\% had the radial access site used). Complications of coronary angiography in 2020 were rare and corresponded to the rates reported in previous years (Table I).

The total number of percutaneous coronary intervention $(\mathrm{PCl})$ procedures was 82349 and was lower by $19 \%$ (2130 PCls per 1 million inhabitants per year) than reported to the ORPKI database in 2019 (Figure 1). The majority of the procedures were done in the setting of 
A

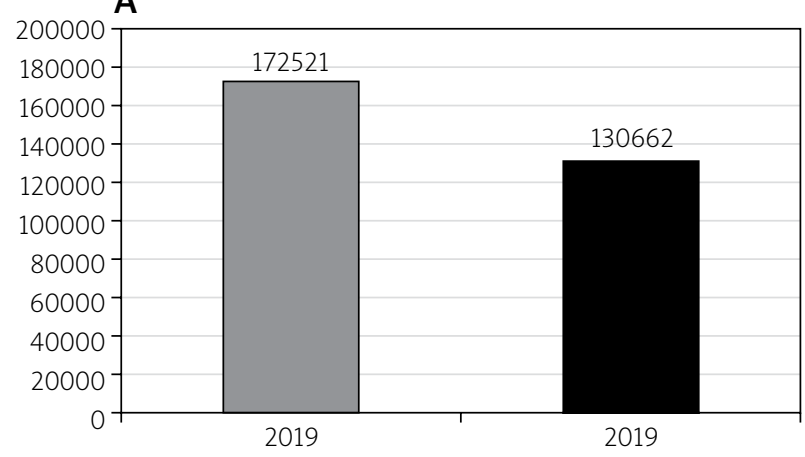

Figure 1. The number of coronary angiography $(A)$ and $\mathrm{PCI}$

Table I. Complications of coronary angiography in Poland in 2020

\begin{tabular}{lcc} 
Parameter & $\%$ & In comparison to 2019 \\
\hline Death & $0.27 \%$ & $\uparrow$ \\
\hline Stroke & $0.01 \%$ & $\leftrightarrow$ \\
\hline Major bleeding at access site & $0.04 \%$ & $\leftrightarrow$ \\
\hline Sudden cardiac arrest & $0.17 \%$ & $\leftrightarrow$ \\
\hline Allergic reaction & $0.01 \%$ & $\leftrightarrow$
\end{tabular}

Table II. Complications of $\mathrm{PCl}$ in Poland in 2020

\begin{tabular}{lcc} 
Parameter & $\%$ & $\begin{array}{c}\text { In comparison } \\
\text { to } 2019\end{array}$ \\
\hline Death: & 0.39 & $\uparrow$ \\
\hline STEMI & 1.01 & \\
\hline NSTEMI & 0.36 & \\
\hline UA & 0.11 & \\
\hline SA & 0.05 & \\
\hline Myocardial infarction & 0.10 & $\downarrow$ \\
\hline Major bleeding from access site & 0.12 & $\leftrightarrow$ \\
\hline Sudden cardiac arrest & 0.38 & $\leftrightarrow$ \\
\hline Allergic reaction & 0.02 & $\uparrow$ \\
\hline Artery perforation & 0.24 & $\leftrightarrow$ \\
\hline No reflow & 0.68 & $\leftrightarrow$
\end{tabular}

Table III. Additional intracoronary assessment in 2020 during angiography and $\mathrm{PCl}$

\begin{tabular}{lccc} 
Variable & $\boldsymbol{N}$ & $\begin{array}{c}\text { \% of all angio } \\
\text { and PCl }\end{array}$ & $\begin{array}{c}\text { Change \% } \\
\text { from 2019 }\end{array}$ \\
\hline FFR & 8420 & 10 & $\downarrow 15$ \\
\hline IVUS & 3923 & 4.8 & $\downarrow 11$ \\
\hline OCT & 239 & 0.3 & $\downarrow 37$
\end{tabular}

ACS: $42 \%$ acute myocardial infarction (21\% STEMI and $21 \%$ NSTEMI), $24 \%$ unstable angina and the remaining $34 \%$ for stable angina. The number of primary PCls per 1 million inhabitants per year is currently 440. There were 16949 PCls in STEMI (13\% decrease) and 17131 in NSTEMI (15\% decrease). Drug-eluting stents (DES) are used in $99.8 \%$ of all cases, which corresponds to 70597

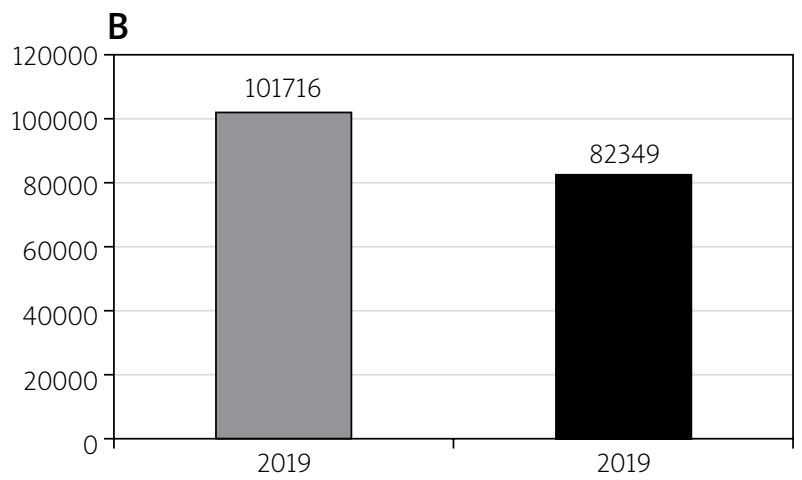

(B) procedures in Poland in the years 2019-2020

stents. Rotablation was used in 1009 procedures $(1.2 \%$ of PCls) - a 14\% decrease in comparison to 2019. Aspiration thrombectomy was used in only 1525 STEMI (9\% of all PCIs in STEMI) cases. We observed a slight increase in the use of guideline-recommended ticagrelor as an adjunct pharmacotherapy both for STEMI (55\%) and NSTEMI (31\%) with the use of prasugrel less than $3 \%$ in either indication. In terms of complex procedures, the number of chronic total occlusion (CTO) procedures was 1708 (26\% decrease) with TIMI 3 flow after the procedure in $67 \%$ of cases. $\mathrm{PCl}$ complications are still rare and are presented in Table II. During the COVID-19 pandemic $0.6 \%$ of all angiographies and $0.4 \%$ of all PCIs were performed in SARS-CoV2 positive patients.

A substantial decrease in the use of adjunctive imaging and diagnostic procedures during $C A G$ and $\mathrm{PCl}$ was observed in 2020 vs. 2019 (Table III).

Structural procedures are a growing part of interventional cardiology in Poland and mainly performed by certified interventional cardiologists. Following recommendations of the European Society of Cardiology, 25 multidisciplinary Heart Teams involving 32 certified interventional cardiologists were established in all 25 transcatheter aortic valve implantation (TAVI) centers. There were $1647 \mathrm{TAVI}$ procedures performed in 25 centers (more than 95\% from femoral access). The median number of TAVI procedures per center per annum decreased to 66 in 2020 due to the opening of 3 new low volume centers. Evolut/Evolut Pro (Medtronic, USA) was implanted in 912 patients, Acurate (Boston Scientific, USA) in 258, Sapien 3/Sapien 3 Ultra/Sapien XT (Edwards Lifesciences, USA) in 243, Portico (Abbott, USA) in 122, MyValve (Merill, India) in 88, Lotus Edge (Boston Scientific, USA) in 19 patients, and Hydra (Vascular Innovations Company, Thailand) in 5.

There were 27 procedures of pulmonary valve implantation including Melody (Medtronic, USA) in 18 and Sapien 3/Sapien XT (Edwards Lifesciences, USA) in 9 patients. Two Melody valves were also implanted in the mitral position.

Only 81 TAVI procedures (4.9\%) were performed using a cerebral protection system - including the commercially available Sentinel system (Boston Scientific, USA) in 73 patients and in 13 patients using ProtEmbo (Protembis, Germany) as part of a scientific study. 


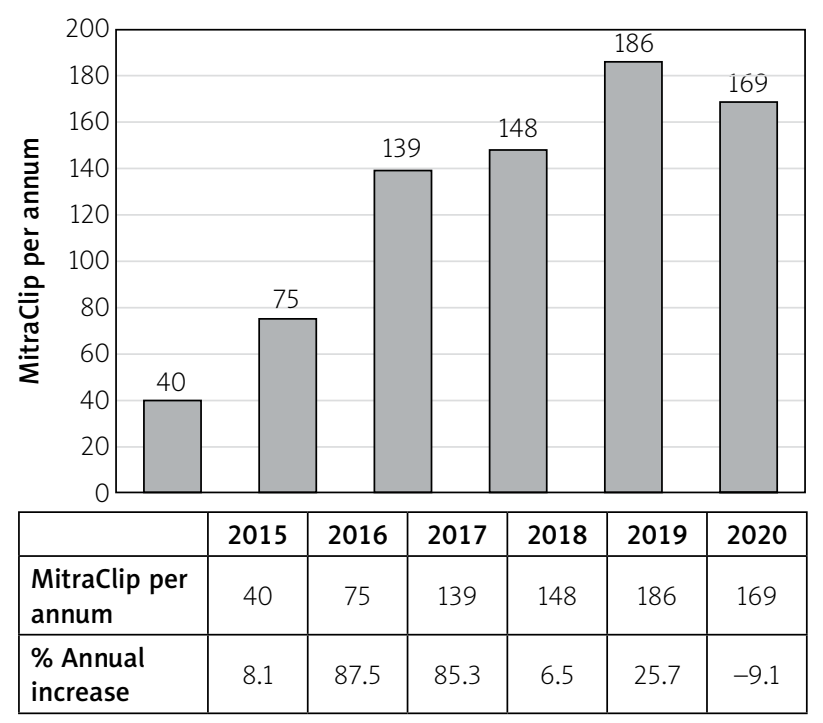

Figure 2. The number of coronary angiography procedures in Poland in consecutive months in 2020

Although the total number of TAVI slightly increased by 107 procedures when compared to 2019 (6.5\% increase year to year), Poland with the number of 43.3 TAVI procedures per million inhabitants in 2020 is far below the European average. In 2017 the annual number of TAVI procedures in Germany reached almost 20000 , with the TAVI rate of 250 per million inhabitants [6]. The data presented at the EuroPCR and ESC events in 2019 confirmed an increase of the number TAVI procedures in France from 7,500 in 2014 to 20,000 in 2018 (137\%) with the TAVI penetration of 193 per million inhabitants in 2018 [7]. Then finally Portugal, often compared to Poland, recorded a significant increase of TAVI procedures during the last 5 years from 29 in 2015 to 72 in 2019 per million inhabitants [8]. It is worth mentioning that all three countries participate in the Valve-for-Life Initiative of the EAPCI ESC (Poland and France since 2015 and Portugal since 2017).

MitraClips (Abbott, USA) were implanted in 15 centers and 169 patients (decrease by $10 \%$ ) mostly due to problems with reimbursement but also COVID restrictions. Moreover, it should be mentioned that Poland with about 4.4 MitraClip procedures per million inhabitants is far below the clinical needs. The dynamics of TAVI and MitraClip procedures in Poland in the last 5 years are presented in Figures 2 and 3.

Only 459 left atrial appendage closure procedures were performed in 28 centers including 236 Watchman/Watchman FLX (Boston Scientific, USA), 221 Amulet (Abbott, USA) and 2 Lambre (Lifetech, China). The number of left atrial appendage (LAA) closure procedures decreased by almost $23 \%$ (year to year) mostly due to COVID restrictions.

Recent studies and changes in guidelines have established the role of patent foramen ovale (PFO) closure in routine practice as a secondary prevention in patients

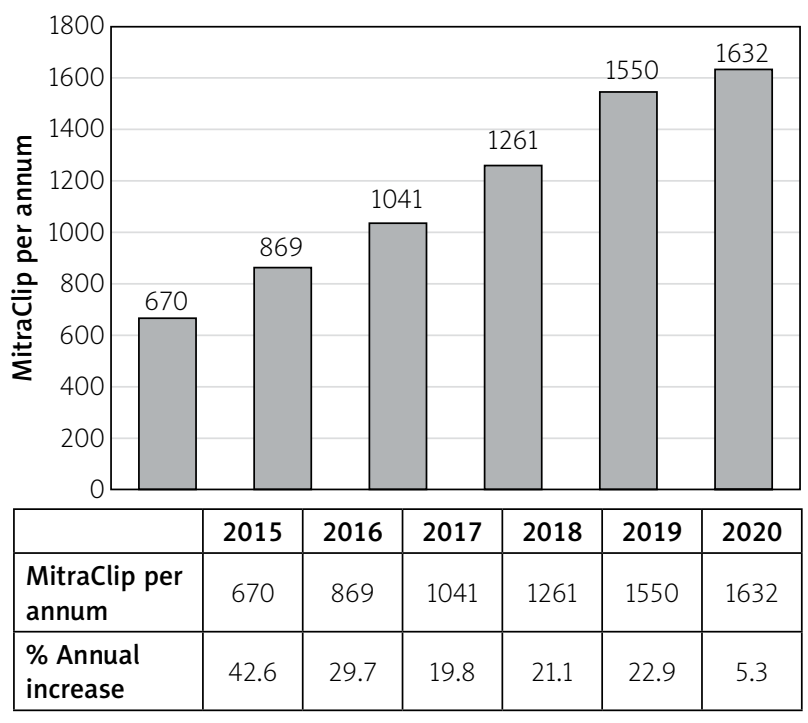

Figure 3. The number of TAVI and MitraClip procedures in Poland in the last 5 years

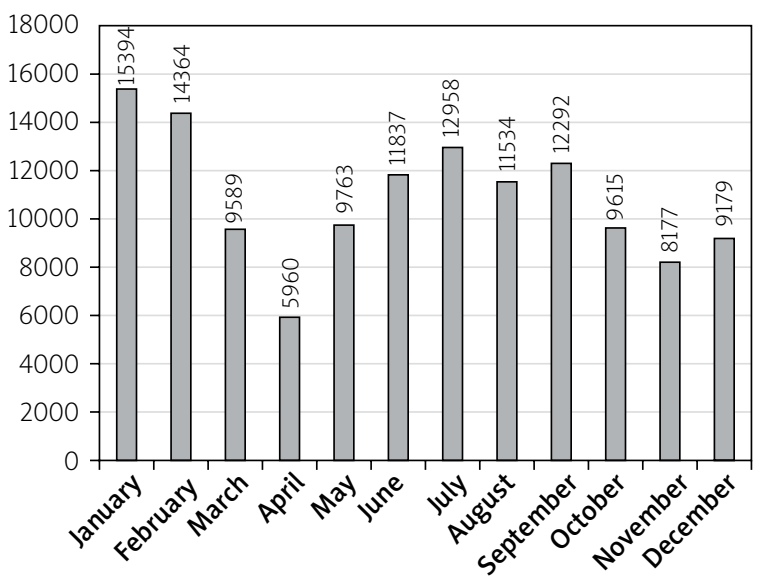

Figure 4. Monthly number of coronary angiographies per month in 2020

after embolic stroke of unknown source not only among cardiologists but also neurologists - 710 PFO closure procedures were performed last year. Additionally, 435 secundum atrial septal defect (ASD II) closure procedures were performed in 2020 by interventional cardiologists.

The ORPKI database was the source material for $20 \mathrm{ma}$ nuscripts published in PubMed in 2019-2020. The AISN PTK issued 1 statement and expert opinion. The scientific activity was enriched by 8 conferences with AISN PTK endorsement and 5 webinars. There were altogether 1 prize and 2 grants on behalf of the AISN PTK in 2020. Many leading Polish cardiologists are active members of EAPCI ESC committees during the 2020-2022 term of Professor Dariusz Dudek as President. There are 14 Polish members of committees with 5 of them serving as Chairs and Co-Chairs (Maciej Lesiak, Wojciech Wojakowski, Marek Grygier, Radosław Parma, Paweł Gąsior). 
In conclusion, the COVID-19 pandemic has had an immense impact on interventional cardiology in Poland. We have observed a substantial decrease in the number of CAG and PCls across various indications as well as underutilization of modern imaging and physiologic assessment techniques. Even though the absolute numbers represent only participating centers, the relative decrease is widely seen also in data provided by the national health insurance provider (NFZ). On the other hand, the use of a radial approach and ticagrelor in $\mathrm{MI}$ patients is well in line with ESC recommendations. It seems that the first wave lockdown had the most devastating impact (March-April 2020) on the overall numbers for 2020 [9] (Figure 4). The number of the majority of structural procedures showed a significant decrease last year, partially because of COVID restrictions (LAA closure, ASD, PFO, MitraClip), limited referrals and even closure of some interventional cardiology sites for non-COVID patients but mostly due to very limited reimbursement (MitraClip). Although the number of TAVI procedures in 2020 increased slightly even despite COVID restrictions (in some centers due to those restrictions and moving patients from classical aortic valve replacement to less invasive TAVI requiring less hospital resources), one should remember that numbers of TAVI and especially MitraClip procedures are far below the European average and current clinical needs in Poland.

\section{Conflict of interest}

The authors declare no conflict of interest.

\section{References}

1. Dudek D, Siudak Z, Grygier M, et al. Interventional cardiology procedures in Poland in 2018. Summary report of the Association of Cardiovascular Interventions of the Polish Cardiac Society (AISN PTK) and Jagiellonian University Medical College. Adv Interv Cardiol 2019; 15: 391-3.

2. Kleczyński P, Siudak Z, Dziewierz A, et al. The network of invasive cardiology facilities in Poland in 2016 (data from the ORPKI Polish National Registry). Kardiol Pol 2018; 76: 805-7.

3. Zabojszcz M, Januszek R, Siudak Z, et al. Association between the mortality rate and operator volume in patients undergoing emergency or elective percutaneous coronary interventions. Kardiol Pol 2020; 78: 138-46.

4. Tokarek T, Dziewierz A, Plens K, et al. Radial approach expertise and clinical outcomes of percutanous coronary interventions performed using femoral approach. J Clin Med 2019; 8: pii: E1484.

5. Januszek R, Siudak Z, Reczuch K, et al. Current trends and procedural outcomes in the era of rotational atherectomy expansion in Poland in the period 2014-2017 (based on the nationwide ORPKI registry). Adv Interv Cardiol 2019; 15: 158-66.

6. Eggebrecht $\mathrm{H}$, Mehta $\mathrm{RH}$. Transcatheter aortic valve implantation (TAVI) in Germany: more than 100,000 procedures and now the standard of care for the elderly. Eurolntervention 2019; 14: e1549-52.
7. https://www.escardio.org/Sub-specialty-communities/European-Association-of-Percutaneous-Cardiovascular-Interventions-(EAPCI)/Advocacy/valve-for-life-france.

8. https://www.escardio.org/Sub-specialty-communities/European-Association-of-Percutaneous-Cardiovascular-Interventions-(EAPCI)/Advocacy/valves-for-life-portugal

9. Siudak Z, Grygier M, Wojakowski W, et al. Clinical and procedural characteristics of COVID-19 patients treated with percutaneous coronary interventions. Catheter Cardiovasc Interv 2020; 96: E568-75. 